\title{
Predictor of Mortality in Acquired Immunodeficiency Syndrome Patients with Central Nervous System Opportunistic Infections
}

\author{
Agustina Lestari, ${ }^{1}$ Sofiati Dian, ${ }^{2}$ Chrysanti Murad $^{3}$ \\ ${ }^{1}$ Faculty of Medicine, Universitas Padjadjaran, ${ }^{2}$ Departement of Physiology Faculty of Medicine \\ Universitas Padjadjaran, ${ }^{3}$ Departement of Physical and Rehabilitation Medicine Faculty of \\ Medicine Universitas Padjadjaran/Dr. Hasan Sadikin General Hospital Bandung
}

\begin{abstract}
Background: Indonesia's increase of acquired immunodeficiency syndrome (AIDS) cases is one of the fastest. Mortality cases of AIDS also increase per year. Central nervous system (CNS) opportunistic infection is one of the most likely manifestations on advanced stage of Human immunodeficiency virus (HIV)/AIDS patients. Common CNS opportunistic infections are toxoplasma infection and tuberculous meningitis (TBM). The study aimed to analyze the predictor of mortality in AIDS patients with CNS opportunistic infections.

Methods: This study reviewed 151 medical records from AIDS patients with CNS opportunistic infection admitted to the Department of Neurology, Dr. Hasan Sadikin General Hospital Bandung from 2007-2012. This study was conducted from April to November 2013. Patients' clinical manifestations (seizure, headache, altered consciousness), laboratory examination (CD4+ level count) and treatment history (antiretroviral or ART and cotrimoxazole) were collected. Chi-square and logistic regression test were used to determine the mortality predictor in patients.

Results: Mortality rate was 37.7\%. Clinical manifestations from patients were seizure $29.8 \%$, altered consciousness $66.2 \%$, and headache $88.7 \%$. Patients had cotrimoxazole treatment $44.4 \%$ and $38.4 \%$ patients had ART. CD4+ level count data from 86 patients were obtained, 94\% had CD4+ level count $\leq 200$. Bivariate analysis showed altered consciousness had significant mortality predictor (Odd ratio (OR): 29.944; 95\% Confidence interval (CI) 6.9-129.945; $\mathrm{p}<0,0$ ). Multivariate analysis showed ART had highest predictive mortality value (OR: 2.968; 95\% CI 1.236-7.126; $\mathrm{p}=0.015$ ).
\end{abstract}

Conclusions: Altered consciousness and no antiretroviral treatment are mortality predictors in AIDS patients with CNS opportunistic infections. [AMJ.2016;3(4):577-82]

Keywords: Acquired immunodeficiency syndrome, CNS opportunistic infection, mortality, predictor

\section{Introduction}

Human Immunodificiency Virus (HIV)/ Acquired immunodeficiency syndrome (AIDS) is one of the few diseases in which complete treatment is still elusive although prevention and treatment to control the virus have been well established for decades. ${ }^{1}$ In the last 30 years, data from the World Health Organization (WHO) and Joint United Nations Programme on HIV/AIDS (UNAIDS) showed that HIV infection caused 25 million deaths around the world. Not like any other countries whose access to antriretroviral (ART) treatment have reduced the number of AIDS related to death cases, reports from WHO and UNAIDS database, in Indonesia from 2006-2009 was recorded an increase in AIDS related to death as much as 3 times along with more than 2 times increase in new HIV infection cases from 2010 to 2011.

With the advanced of science, it was known that the two main targets of HIV infection are the limphoid system and central nervous system (CNS), ${ }^{2,3}$ Infection of HIV markedly affected immune system was shown with immunodeficiency and level of CD4+ T cell, which made someone prone to opportunistic infection, neoplasm, and many other clinical conditions. ${ }^{2}$ From numerous earlier research in developing countries, the main cause of CNS impairment among HIV/AIDS patients were CNS opportunistic infections. ${ }^{4}$

Severe immunodeficiency in a late phase of HIV infection with high virus load both on systemic and CNS has a positive correlation

Correspondence: Agustina Lestari, Faculty of Medicine, Universitas Padjadjaran, Jalan Raya Bandung-Sumedang Km.21, Jatinangor, Sumedang, Indonesia, Phone: +62 8172375599 Email: agustina.lestari@gmail.com 
with the likelihood of CNS opportunistic infection. Environment and access to ART become important factors to the variation of CNS opportunistic infection between developed and developing countries. ${ }^{5}$

The common CNS opportunistic infections in HIV/AIDS patients are toxoplasma infection, tuberculous meningitis (TBM), cryptococcus infection, progressive multifocal leukoencelopathy (PML) and primary CNS lymphoma. ${ }^{2,6}$ The risk of CNS opportunistic infection is different according to patient's CD4+ T cell level; patients with CD4+ T cell level $200-500 / \mu \mathrm{L}$ have risk being infected by tuberculous meningitis, patients with $\mathrm{CD} 4+$ $\mathrm{T}$ cell level $<200 / \mu \mathrm{L}$ have risk being infected by toxoplasmosis encephalitis, cryptococcus meningitis, cytomegalovirus (CMV) infection, primary lymphoma CNS, and PML. ${ }^{5}$ Almost all CNS opportunistic infections originate from activated latent pathogen, except PML that could be caused by direct HIV-1 virus infection. ${ }^{5}$ Most of all CNS opportunistic infections do not have specific symptoms like fever or lethargic. Symptoms which usually arise are headache, seizure, altered consciousness, altered mental function, focal deficit and cranial neuropathy. ${ }^{5}$

A study in Bangkok Thailand $d^{5}$, showed that mortality rate of HIV/AIDS patients with CNS opportunistic infections was 14.9\% and in Nigeria $^{7}$ and Ethiopia ${ }^{4}$ showed high case-fatality up to $45 \%$. There were several predictors of mortality in HIV/AIDS cases. The study in Ethiophia ${ }^{4}$, discovered that the area of the patients' residency, altered consciousness, and seizure were predictors of mortality in HIV/AIDS cases.

Based on these evidences, the objective of this study was to analyze the predictors of mortality in Acquired Immunodeficiency Syndrome patients with central nervous system opportunistic infections in Bandung, Indonesia.

\section{Methods}

A retrospective cross-sectional study was conducted at Dr. Hasan Sadikin General Hospital, Bandung, Indonesia from April to November 2013. Data were obtained from medical records of AIDS patients admitted to the Neurology Department of Dr. Hasan Sadikin General Hospital from January 2007-December 2012. The inclusion criteria were medical records of AIDS patients with CNS opportunistic infection(s) consisting of information of altered consciousness, seizure, headache, CD4+ level count, ART treatment, cotrimoxazole treatment and the outcome of the treatment. The exclusion criteria were imcomplete information in the patient's medical records and unclear patient's cause of death.

From 2007 to 2012, there were 153 cases of AIDS with CNS opportunistic infections from 342 cases of HIV/AIDS, but only 151 cases met the inclusion criteria.

The collected data were analyzed statistically using the Chi-square test for bivariate analysis and logistic regression for multivariate analysis with $p$ value $<0.05$ and $95 \%$ confidence interval as statistical significance. The Ethical approval for this study was obtained from the Health Research Ethics Committee of Faculty of Medicine of Universits Padjadjaran/Dr. Hasan Sadikin General Hospital.

\section{Results}

This study discovered that male had

Table 1 Characteristics of Patients

\begin{tabular}{|c|c|}
\hline Variable & $n=151$ \\
\hline \multicolumn{2}{|l|}{ Sex } \\
\hline Female & $42(28.1 \%)$ \\
\hline Male & 109 (71.9\%) \\
\hline \multicolumn{2}{|l|}{ Age (years old) } \\
\hline$<30$ & $63(41.7 \%)$ \\
\hline$\geq 30$ & 88 (58.3\%) \\
\hline Mean & 30.8 \\
\hline Range & 20-54 \\
\hline Mortality rate & 57 (37.7\%) \\
\hline
\end{tabular}


Table 2 Distribution of CNS Opportunistic Infections

\begin{tabular}{lc}
\hline \multicolumn{1}{c}{ CNS opportunistic infections } & Total (n) \\
\hline Cerebral toxoplasmosis, TBM, Criptococcus meningitis, Tuberculoma & 1 \\
Cerebral toxoplasmosis, TBM, Tuberculoma & 1 \\
Cerebral toxoplasmosis, Tuberculoma & 19 \\
Cerebral toxoplasmosis, Criptococcus meningitis, Tuberculoma & 1 \\
Cerebral toxoplasmosis, TBM, Criptococcus meningitis & 1 \\
Cerebral toxoplasmosis, Criptococcus meningitis & 2 \\
Cerebral toxoplasmosis, TBM, CMV infection & 1 \\
Cerebral toxoplasmosis, TBM & 8 \\
Cerebral toxoplasmosis, CMV infection & 2 \\
Cerebral toxoplasmosis & 57 \\
TBM, Criptococcus meningitis & 6 \\
TBM, Tuberculoma & 2 \\
TBM & 39 \\
Criptococcus meningitis & 5 \\
Criptococcus meningitis, Tuberculoma, & 1 \\
Tuberculoma & 5 \\
\hline
\end{tabular}

Note: TBM= Tuberculous meningitis; CMV=Cytomegalovirus

higher percentage of AIDS cases with CNS opportunistic infections compared to female. Morover, those cases occurred mostly to young people although the age range was wide. From all the cases, mortality was still high (Table 1). In terms of CNS opportunistic infections, most of the patients (106 cases) had only 1 infection. On the other hand, there were patients with multiple infections. One of the patients had 4 infections, 4 patients had 3 infections, and 40 patients had 2 infections. (Table 2). The most infection occured in those patients was cerebral toxoplasmosis 93 (61.6\%). Other infections occured were TBM, followed by tuberculoma, cryptococcus meningitis and CMV infection (39.1\%, 20.0\%, 11.3\%, 2.0\%, respectively) (Table 3 ).

There were 86 recorded data for $\mathrm{CD} 4+$ level count: 81 patients $(94,20 \%)$ had CD4+ level count $\leq 200$ and 5 patients $(5,80 \%)$ had CD $4+$ level count $>200$ (Table 3 ).

Furthermore, there was $29.8 \%$ patients with seizure; $66.2 \%$ patients with altered consciousness; $88.7 \%$ patients with headache; $44.4 \%$ patients with cotrimoxazole treatment; and $38.4 \%$ patients with antiretroviral treatment (Table 4).

The highest case of fatality rateswas tuberculoma (60\%), followed by cryptococcus meningitis (58.8\%), TBM (47.5\%), cerebral toxoplasmosis $(35.5 \%)$, and CMV infection (33.3\%) respectively (Table 4 ).

The bivariate statistical analysis was performed to determine which variables werecorrelated to mortality. This study discovered that altered consciousness was the predictor of mortality AIDS patients with CNS opportunistic infection. Other variables, such as seizure, headache, CD4+ level count, cotrimoxazole treatment were not predictors of mortality in these cases ( $p$ value $\geq 0.05$. Although no ART treatment was significantly related to predictor of mortality in AIDS patients with CNS opportunistic infections, the OR was below 1 (Table 5).

Moreover, the logistic regression analysis was performed to determine which variable was the strongest predictor among the variables. The logistic regression analysis was performed for five variables (seizure, altered consciousness, headache, cotrimoxazole and ART). The variable CD4+ level count was not included into the analysis because the number of medical records that had the CD4+ level count information was only 86 medical records.

The study showed that no treatment by ART (OR=2.968; 95\% CI 1.236-7.126; p=0.015) was predictor for mortality AIDS patient with CNS opportunistic infection. Patients who 
Table 3 Demographic and Laboratory Findings per Infection

\begin{tabular}{lccc}
\hline CNS Opportunistic Infections & Age (years old) & Sex & CD4+ level \\
\hline TBM(59) & $<30: 29(49.2 \%)$ & Male : $37(62.7 \%)$ & $\leq 200: 28(93.3 \%)$ \\
& $\geq 30: 30(50.8 \%)$ & Female : $22(37.3 \%)$ & $>200: 2(6.7 \%)$ \\
Cryptococcus Meningitis (17) & $<30: 7(41.2 \%)$ & Male : $13(76.5 \%)$ & $\leq 200: 17(100 \%)$ \\
& $\geq 30: 10(58.8 \%)$ & Female : $4(23.5 \%)$ & $>200: 0(0 \%)$ \\
Cerebral Toxoplasmosis (93) & $<30: 36(38.7 \%)$ & Male : $69(74.2 \%)$ & $\leq 200: 53(94.6 \%)$ \\
& $<30: 11(36.7 \%)$ & Male : $18(60.0 \%)$ & $\leq 200: 13(92.9 \%)$ \\
Tuberculoma (30) & $\geq 30: 19(63.3 \%)$ & Female : $12(40.0 \%)$ & $>200: 1(7.1 \%)$ \\
CMV (3) & $<30: 0(0 \%)$ & Male : $2(66.7 \%)$ & $\leq 200: 3(100 \%)$ \\
& $\geq 30: 3(100 \%)$ & Female : $1(33.3 \%)$ & $>200:-$ \\
\hline
\end{tabular}

never had ART treatment had a mortality risk 2.968 times higher compared to patients who had ART treatment. Although altered consciousness was significantly related to predictor of mortality in AIDS patients with CNS opportunistic infections, the OR was below 1 (Table 6).

\section{Discussion}

This study discovered that the mortality of AIDS patients with CNS opportunistic infections was higher that the mortality in Bangkok Thailand. ${ }^{8}$ It was suspected that this condition occured because Thailand had a good treatment protocol regarding HIV patients and also the level of the people's and the government's awareness about this disease was higher compared to Indonesia. On the other hand, the mortality rate in this study was lower compared to a study in Ethiopia, Africa $^{4}$ as this region had higher HIV/AIDS cases prevalence compared to Indonesia.

Besides, the sex distribution showed that male outnumbered female and the majority of patients were at young age (mean age 30.8 years old). These findings were similar with several previous studies. ${ }^{4,7,9}$

Interestingly, compared to a study in Thailand ${ }^{10}$ which stated that cryptococcal meningitis was the most frequent AIDS-related opportunistic infection; in this study, cerebral toxoplasmosis had the highest prevalence among other infections. Moreover, another study in Indonesia ${ }^{11}$ stated that cryptococcal meningitis was the most important CNS opportunistic infection. Cryptococcal meningitis arises on patients with very low CD4+ level count and usually patients died before they are diagnosed for the disease. HIV patients often seek care only when they are already on their late stage and low awareness to follow up especially the measurement of their CD4+ level count. The percentage of cerebral toxoplasmosis in this study was higher compared with a study in Ethiopia ${ }^{4}$ $(36.6 \%)$ and a study in India ${ }^{12}(8.8 \%)$. This may be caused by the difference in the prophylaxis treatment protocol especially cotrimoxazole in Indonesia and other countries.

Table 4 Clinical Manifestation, Laboratory Findings and Treatment per Infection

\begin{tabular}{lccccc}
\hline CNS Opportunistic Infections & Death & $\begin{array}{c}\text { Altered } \\
\text { Conciousness }\end{array}$ & Headache & Cotrimoxazole & ART \\
\hline TBM (59) & $28(47.5 \%)$ & $44(74.6 \%)$ & $52(88.1 \%)$ & $25(42.4 \%)$ & $16(27.1 \%)$ \\
Cryptococcus Meningitis (17) & $10(58.8 \%)$ & $12(70.6 \%)$ & $17(100 \%)$ & $7(41.2 \%)$ & $8(47.1 \%)$ \\
Cerebral Toxoplasmosis (93) & $33(35.5 \%)$ & $62(66.7 \%)$ & $81(87.1 \%)$ & $44(47.3 \%)$ & $39(41.9 \%)$ \\
Tuberculoma (30) & $18(60.0 \%)$ & $20(66.7 \%)$ & $26(86.7 \%)$ & $15(50.0 \%)$ & $8(26.7 \%)$ \\
CMV (3) & $1(33.3 \%)$ & $1(33.3 \%)$ & $2(66.7 \%)$ & $1(33.3 \%)$ & $2(66.7 \%)$ \\
\hline
\end{tabular}


Table 5 Bivariate (Chi-square) Analysis

\begin{tabular}{|c|c|c|c|c|}
\hline Risk Factor & & Total (57) & OR $(95 \% \mathrm{CI})$ & p \\
\hline Seizure & & 20 & $1.492(0.733-3.037)$ & 0.269 \\
\hline Altered Consciousness & & 55 & $29.944(6,900-129.945)$ & 0.0 \\
\hline Headache & & 51 & $1.127(0.393-3.232)$ & 0.825 \\
\hline \multirow[t]{2}{*}{ CD4+level count (28) } & $>200$ & 2 & 1 & 0.714 \\
\hline & $\leq 200$ & 26 & $1.410(0.222-8.960)$ & \\
\hline Cotrimoxazole treatment & & 26 & $1.084(0.559-2.101)$ & 0.811 \\
\hline ART treatment & & 45 & $0.278(0.131-0.592)$ & 0.001 \\
\hline
\end{tabular}

Table 6 Multivariate (Logistic Regression) Analysis

\begin{tabular}{lccc}
\hline \multicolumn{1}{c}{ Risk Factor } & Total (57) & OR $\mathbf{( 9 5 \% ~ C I ) ~}$ & p \\
\hline Seizure & 20 & $1.228(0.536-2.814)$ & 0.628 \\
Altered Consciousness & 55 & $0.037(0.008-0.163)$ & 0.000 \\
Headache & 51 & $0.661(0.187-2.335)$ & 0.661 \\
Cotrimoxazole & 26 & $0.718(0.320-1.612)$ & 0.422 \\
ART & 45 & $2.968(1.236-7.126)$ & 0.015 \\
\hline
\end{tabular}

Note $:$ OR $=$ Odd ratio $; \mathrm{CI}=$ Confidence Interval; $\mathrm{ART}=$ Anti retrovirus

Other infections identified in this study were TBM, tuberculoma, cryptococcus meningitis and CMV infection that were similar with the findings of other studies ${ }^{4,12,13}$, except a study in The United States ${ }^{4}$ where TBM and bacterial meningitis were rarely found. ${ }^{4}$ This different result showed that environment and access to ART are important factors to CNS opportunistic infection variations. ${ }^{5}$

In addition, headache was identified as the highest prevalence of clinical symptoms, followed by altered consciousness and seizure. These findings were similar with a study in Ethiopia ${ }^{4}$.

From 86 patients, the majority $(94,20 \%)$ had CD4+ level count $\leq 200$ and only $5,80 \%$ had CD4+ level count $>200$, the result showed consistency with studies in Ethiopia and India. ${ }^{4,12}$ No patients with cryptococcus meningitis and CMV infections had CD4+ level count $>200$. This was in line with HIVassociated opportunistic infections of the CNS literatur. ${ }^{5}$

A total of $43.8 \%$ patients had a history of cotrimoxazole prophylaxis treatment or the treatment was given during admission. Out of 57 mortality cases, $54.4 \%$ patients never had a cotrimoxazole treatment and it was higher compared to the study in Ethiopia ${ }^{4}(44.6 \%)$. Prior to this, early detection of HIV and early cotrimoxazole prophylaxis treatment as primary prophylaxis on HIV/AIDS patients may suggest a decrease in the prevalence cerebral toxoplasmosis and its mortality. Thirty seven point nine percent patients had ART treatment both before and after admission. Among the mortality cases, $78.9 \%$ patients had no ART treatment. This was much higher than the study in Ethiopia (46.1\%). The data support Indonesia's need for more effective prevention and detection of HIV infection as well as access to ART treatment to improve cases of AIDS related to death. ${ }^{5,11}$

The bivariate analysis result showed altered consciousness (OR=29.944; 95\% CI 6.900129.945; $\mathrm{p}=0.0$ ) as predictor and multivariate analysis showed ART (OR=2.968; 95\% CI 1.236-7.126; $\mathrm{p}=0.015)$ as predictor. The multivariate analysis calculated 5 variables, to find which variable had the strongest relation to mortality cases. Our study showed altered consciousness was the predictor mortality dependently and ART treatment was the strongest predictor among all variables. The used of ART was a predictor mortality for AIDS patients with CNS opportunistic infection. Patients who never had ART treatment had more mortality risk than patients who had ART treatment. Decrease immune system markedly showed stage severity of the disease and make 
someone prone to opportunistic infection. ${ }^{2}$ Antiretroviral treatment has functioned as disease severity suppressor to prevent the disease to an advanced stage and prevent opportunistic infections by increase immune system. ${ }^{2}$ In a future study, analysis about ART treatment and CD4+ level count could be added to investigate more exact about the correlation between ART treatment, CD4+ level count and disease severity and its outcome.

This study had several limitations. Both cotrimoxazole and ART treatment were undifferentiated before and after admission. The study did not investigate the time of HIV infection confirmed diagnosis and multiple CNS opportunistic infections that may affect patient's outcome and mortality. Medical records data recording could be improved to provide more complete data, especially for laboratory examination result such as CD4+ count level to support a better research in the future.

In conclusion, the majority of the patients have CD4+ count level $\leq 200$ and only onethird of the patients have ART treatment. Altered conciousness and no ART treatment are suspected to be the predictor of mortality in AIDS patients with CNS opportunistic infections. The improvement of HIV screening, earlier primary prophylaxis treatment, access to ART and follow up of CD4+ count level will result into a better outcome.

\section{References}

1. Hoffmann C, Rockstroh JK, Kamps BS, editors. HIV medicine 2007. 15th ed. Philadelphia. Flying Publisher; 2007.

2. Anthony FS, Clifford HL. Human immunodeficiency virus disease: AIDS and related disorders. Dan LL. Harrion's principle of internal medicine. 18th ed. Alabama. Mc Graw Hill; 2012. p. 1506-87.

3. Vinay K, Abul AK, Nelson F, Jon AC. Robbin and Cotran pathologic basics of disease 8th ed. Philadelphia : Saunders Elsevier; 2010.

4. Berhe T, Melkamul Y, Amare A. The pattern and predictors of mortality of HIV/AIDS patients with neurologic manifestation in
Ethiopia: a retrospective study. AIDS Res Ther. 2012;9:11.

5. Tan IL, Smith BR, van Geldern G, Mateen FJ, McArthur JC. HIV-associated opportunistic infections of the CNS. Lancet Neurol. 2012;11(7):605-17.

6. Barley BC. Acquired immunodeficiency syndrome (AIDS). In: Lewis RP, editor. Merritt's neurology. 11th ed. Philadelphia USA: Lippincot Williams \& Wilkins; 2005. p. 211-27.

7. Ogun SA, Ojini F, Okubadejo N, Danesi M, Kolapo K, Osalusi B, et al. Pattern and outcome of neurological manifestations of HIV/AIDS-a review of 154 cases in a Nigerian University Teaching Hospital-a preliminary report. African Journal of Neurological Sciences. 2005;24(1):29-36.

8. Kongsiriwattanakul S, Suankratay C. Central nervous system infections in HIVinfected patients hospitalized at King Chulalongkorn Memorial Hospital. J Med Assoc Thai. 2011;94(5):551-8.

9. Isezuo SA, Sani AZ, Ezunu E, Maiyaki S, Njoku $\mathrm{CH}$, Obembe A. Clinical neuropathy in HIV/ AIDS: an eight-year review of hospitalized patients in Sokoto, northwestern Nigeria. Trop Doct. 2009;39(3):133-5

10. Kantipong P, Murakami K, Moolphate S, Aung MN, Yamada N. Causes of mortality among tuberculosis and HIV co-infected patients in Chiang Rai, Northern Thailand. HIV AIDS (Auckl). 2012;4:159-68.

11. Ganiem AR, Parwati I, Wisaksana R, van der Zandeh A, van de Beek D, Sturm P, et al. The effect of HIV infection on adult meningitis in Indonesia: a prospective cohort study. AIDS. 2009;23(17):2309-16

12. Satya A, Kumar G, Pratap S, Vivekananda V, Madhukar R, Shyam. Neurological manifestations of HIV- infected patients around Varanasi, India. African Journal of Neurological Sciences. 2006;25(1):33-40

13. Almeida SMd, Ribeiro CE, Luis Felipe Cavalli Pessa, Moreira SDR, Vidal LR, Nogueira $\mathrm{MB}$, et al. Incidence of neurological manifestations as AIDS defining clinical conditions in Brazil. BMC Proceedings. 2008:2:45. 